\title{
Glueballs as gravitons in holographic approaches
}

\author{
M. Rinaldi* \\ Dipartimento di Fisica e Geologia. Università degli studi di Perugia. INFN section of Perugia. \\ Via A. Pascoli, Perugia, Iataly. \\ E-mail: matteo.rinaldi@pg.infn.it
}

In this contribution we present a a phenomenological analysis of the scalar glueball and scalar meson spectra within AdS/QCD models in the bottom-up approach. In particular, we consider a Light-Front QCD framework which allows to relate the AdS/QCD mode functions to the hadronic wave-functions. Such a procedure is here adopted to analyse the mixing between scalar mesons and glueballs.

Light Cone 2019 - QCD on the light cone: from hadrons to heavy ions - LC2019

16-20 September 2019

Ecole Polytechnique, Palaiseau, France

${ }^{*}$ Speaker. 


\section{Introduction}

In this contribution we investigate the structure of glueballs, for which some information on their spectra and properties have been collected in particular within the lattice approach $[1,2,3]$. However, if glueballs exist they would mix with meson states with same quantum numbers making difficult their direct characterization. Here we consider AdS/QCD models to access non perturbative features of glueballs $[4,5]$. The holographic principle relies in a correspondence between a five dimensional classical theory with an AdS metric and a supersymmetric conformal quantum field theory. Since the latter is different from QCD, we consider a bottom-up [6, 7, 8]. It consists in properly modifying the five dimensional classical theory to resemble QCD as much as possible. In this analysis, we used the so called soft-wall ( $\mathrm{SW}$ ) models where a dilaton field is introduced to softly break conformal invariance. This model has been successfully applied to investigate non perturbative features of hadrons and has been extended to study the glueball spectrum [5, 8, 9, 10, 11]. We describe the glueball lattice spectrum by means of the AdS/QCD correspondence and then we compare it with the spectrum of $f_{0}$ 's, experimentally determined [12]. Only when the masses of the glueballs and the mesons are close, mixing is to be expected [13]. However, if this mass condition is associated to a different dynamics, mixing will not happen [14]. Therefore, we are looking for meson and glueball states with similar masses but generated by different dynamics.

\section{Scalar glueball and scalar meson spectrum in a bottom-up approach}

In the botton-up approach on the AdS/CFT, one implements duality in nearly conformal conditions defining QCD on the four dimensional boundary and introducing a bulk space which is a slice of $A d S_{5}$ whose size is related to $\Lambda_{Q C D}[6,7,10]$. The metric of this space can be written as

$$
d s^{2}=g_{M N} d x^{M} d x^{N}=\frac{R^{2}}{z^{2}}\left(d z^{2}+\eta_{\mu v} d x^{\mu} d x^{v}\right)+R^{2} d \Omega_{5},
$$

where $\eta_{\mu \nu}$ is the Minkowski metric. Within the bottom-up SW approach, glueballs arise from the correspondence with a field in $A d S_{5}$ [11]. In our recent work we have described the scalar glueball spectrum as that of a graviton in $A d S_{5}$ with a modified SW metric [5].

\subsection{Scalar glueballs and mesons in the soft wall model}

For a scalar field in the AdS space, see Eq. (2.1) for the adopted metric, within the SW model, the associated mode function can be obtained from the equation of motion (EoM), see e.g. Ref. [9].

$$
-\phi_{\chi}^{\prime \prime}(z)+\left[\kappa^{4} z^{2}+\frac{15-4 M_{5 \chi}^{2} R^{2}}{4 z^{2}}+2 \kappa^{2}\right] \phi_{\chi}(z)=\mu_{\chi}^{2} \phi_{\chi}(z)
$$

where here, $\chi$ identifies a scalar glueball or a meson field ( $\chi=g, m$ respectively), $M_{5 \chi}^{2}$ is the $A d S_{5}$ mass with $M_{5 g}=0$ while for the meson $R^{2} M_{5 m}^{2}=-3$. In the above relation $\mu_{\chi}$ is the mass of the $\chi$ field. The solution of the above equation leads to the a spectrum: $\mu_{n_{\chi}}^{2}=\left[4 n_{\chi}+C_{\chi}\right] \kappa_{\chi}^{2}$, where $n_{\chi}$ represents the mode number and $\kappa_{\chi}$ the mass scale (in principle it could depend on the kind of hadron), $C_{m}=6$ and $C_{g}=8$. The corresponding normalised mode functions can be written as: 


$$
\begin{aligned}
& \Psi_{n_{g}}(z)=\sqrt{\left(n_{g}+1\right)\left(n_{g}+2\right) / 2} e^{-\kappa^{2} z^{2} / 2} z^{5 / 2}{ }_{1} F_{1}\left(-n_{g}, 3, \kappa^{2} z^{2}\right), \\
& \Phi_{n_{m}}(z)=\sqrt{2\left(n_{m}+1\right)} e^{-\kappa z^{2} / 2} z^{3 / 2}{ }_{1} F_{1}\left(-n_{m}, 2, \kappa^{2} z^{2}\right) .
\end{aligned}
$$

\subsection{The softwall-graviton model}

In ref. [5], we discussed the possibility that the glueball field is dual to a graviton, to this we generalised the background metric:

$$
\bar{g}_{M N}=e^{-\alpha^{2} z^{2} / R^{2}} g_{M N}
$$

with $\alpha^{2}<0$ in order to have bound states. The EoM is obtained by solving the Einstein equation for the above metric [5]:

$$
-\Psi^{\prime \prime}(t)+\left[\frac{8 e^{2 t^{2}}}{t^{2}}-\frac{17}{4 t^{2}}+14-15 t^{2}\right] \Psi(t)=\frac{2 \mu^{2}}{a^{2}} \Psi(t),
$$

where here $t=a z / \sqrt{2}$ and $a=i \alpha$, see ref. [5] for details on the spectrum. In the left panel of Fig. 1 we show our fit of the scalar meson and glueball spectra obtained within AdS/QCD models. The fit has been obtained by properly choosing the mass scale energy. In the case of mesons (lower line and dots in left panel of Fig. 1) we find a reasonable result for $\kappa \sim 410 \mathrm{MeV}$ by skipping the $f_{0}(500)$ state which is not a conventional meson state $[15,16]$. For glueballs, the SW model is unable to properly fit the lattice spectrum [5]. Within the graviton model, the best fit is found for $\alpha \sim 370$ $\mathrm{MeV}$, see upper line of the left panel of Fig. 1. In this case, the spectrum has an almost linear behaviour with no softening of the slope in the analysed region. Due to the difference between the slopes of the glueball and meson fits for large mode numbers, the related mode function for a meson will oscillate more than that for a glueball with similar mass. Therefore the large difference in mode numbers could be interpreted as a weak mixing between the statess.
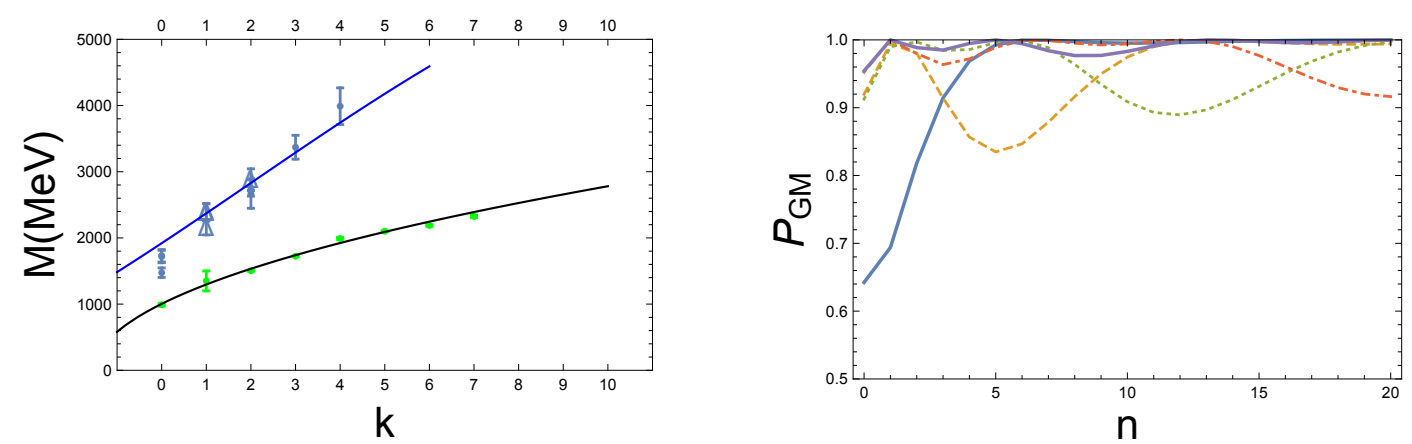

Figure 1: Left panel: fits of glueball spectrum within the graviton model (full upper line) and scalar meson spectrum (full lower line) obtained within the dilaton SW model [17,5]. The dark dots represent glueball spectrum obtained within lattice QCD $[1,2,3]$. The light dots represent the scalar meson spectrum obtained from experimental data [12]. Right Panel: We plot the probability of no mixing for the glueball with mode numbers $n_{g}=0$ (solid), 1 (dashed), 2 (dotted), 3 (dot-dashed), 4 (solid) as a function of meson mode number $n$. 


\section{Glueball-Meson mixing}

Here we show the formalism to look for dynamical regions were mixing is not favorable and therefore states with mostly gluonic valence structure might exist. We consider here an holographic light-fron (LF) representation of the EoM, in $A d S$ space. The latter can be recast in the form of a LF Hamiltonian [6]

$$
H_{L C}\left|\Psi_{k}>=M^{2}\right| \Psi_{k}>
$$

In this equation both quantities are adimensional. We consider a two dimensional Hilbert space generated by a meson and a glueball states, $\left\{\left|\Psi_{m}\right\rangle,\left|\Phi_{g}\right\rangle\right\}$. Mixing occurs when the hamiltonian is not diagonal in the subspace. A matrix representation of the hamiltonian in this subspace is given by

$$
[H]=\left(\begin{array}{cc}
m_{1} & \alpha \\
\alpha & m_{2}
\end{array}\right),
$$

where $\alpha=<\Psi_{m}|H| \Phi_{g}>, m_{1}=<\Psi_{m}|H| \Psi_{m}>$ and $m_{2}=<\Phi_{g}|H| \Phi_{g}>$. We are assuming $m_{2}>m_{1}$ and for simplicity $\alpha$ real and positive. Large $N_{C}$ QCD analysis shows that $m_{1}, m_{2} \sim \mathscr{O}\left(N_{C}^{0}\right)$ and $\alpha \sim \mathscr{O}\left(N_{C}^{-1 / 2}\right)$ [13]. After diagonalization the eigenstates have a mass $M_{ \pm}=m \pm \sqrt{\alpha^{2}+(\Delta m)^{2}}$, where $m=\left(m_{1}+m_{2}\right) / 2$ and $\Delta m=\left(m_{2}-m_{1}\right) / 2$. The first physical meson, assuming to be the lightest one, is given by the eigenvector of $H$, see Ref. [18]. In our fit we have fixed the meson spectrum to the experimental values and therefore $\mid \Psi_{p h y}>$ represents a physical meson state while we have fixed the glueball spectrum to the lattice values, therefore the glueball state is our initial state $\left|\Phi_{g}\right\rangle$, thus

$$
\left|<\Psi_{p h y}\right| \Phi_{g}>\left.\right|^{2}=\frac{\alpha^{2}}{\left(M_{-}-m_{2}\right)^{2}} .
$$

The mixing probability is proportional to the overlap of these two wave functions (w.f.). We consider Eq. (2.3) for the meson w.f. and that from Eq. (2.6) for the glueball one. We calculate the probability for no mixing, i.e., $P_{G M}=1-\left|\left\langle\Psi_{p h y} \mid \Phi_{g}\right\rangle\right|^{2}$. As one can see in the right panel of Fig. 1, the mixing should occur when $n_{g}=2,3,4$ and, following the similar mass condition, for meson mode numbers such as $n \sim 10,13,17$. As one can see in the right panel of Fig. 1 this condition reduces the overlap probability for mixing dramatically. The outcome of our analysis is that the AdS/QCD approach predicts the existence of almost pure glueball states in the scalar sector in the mass range above $2 \mathrm{GeV}$.

\section{Conclusion}

We have performed a phenomenological analysis of the scalar glueball and scalar meson spectrum based on the AdS/QCD correspondence within the SW and softwall-graviton approaches. Theoretical outcomes have been compared with lattice $Q C D$ data in the case of the glueballs and the experimental $f_{0}$ spectrum of the PDG tables in the case of the mesons. Assuming a light-front quantum mechanical description of AdS/QCD correspondence, we have shown that the overlap probability of heavy glueballs to heavy mesons is small and thus one expects little mixing in the high mass sector. Therefore, this is the kinematical region to look for almost pure glueball states. 


\section{Acknowledgments}

The author thanks all the orginzers of the conference for the support given for this talk. M.R. thanks Vicente Vento for discussions. This work was supported in part by the STRONG-2020 project of the European Union's Horizon 2020 research and innovation programme under grant agreement No 824093.

\section{References}

[1] C. J. Morningstar and M. J. Peardon, The Glueball spectrum from an anisotropic lattice study, Phys. Rev. D60 (1999) 034509 [hep-lat/9901004].

[2] Y. Chen et al., Glueball spectrum and matrix elements on anisotropic lattices, Phys. Rev. D73 (2006) 014516 [hep-lat/0510074].

[3] B. Lucini, M. Teper and U. Wenger, Glueballs and k-strings in SU(N) gauge theories: Calculations with improved operators, JHEP 06 (2004) 012 [hep-lat / 0404008 ].

[4] V. Vento, AdS gravity and the scalar glueball spectrum, Eur. Phys. J. A53 (2017) 185 [1706.06811].

[5] M. Rinaldi and V. Vento, Scalar and Tensor Glueballs as Gravitons, Eur. Phys. J. A54 (2018) 151 [1710.09225].

[6] S. J. Brodsky and G. F. de Téramond, Light-front hadron dynamics and AdS/CFT correspondence, Phys. Lett. B582 (2004) 211 [hep-th/0310227].

[7] L. Da Rold and A. Pomarol, Chiral symmetry breaking from five dimensional spaces, Nucl. Phys. B721 (2005) 79 [hep-ph/ 0501218$].$

[8] A. Karch, E. Katz, D. T. Son and M. A. Stephanov, Linear confinement and AdS/QCD, Phys. Rev. D74 (2006) 015005 [hep-ph / 0602229 ].

[9] E. Folco Capossoli and H. Boschi-Filho, Glueball spectra and Regge trajectories from a modified holographic softwall model, Phys. Lett. B753 (2016) 419 [1510 . 03372].

[10] J. Erlich, E. Katz, D. T. Son and M. A. Stephanov, QCD and a holographic model of hadrons, Phys. Rev. Lett. 95 (2005) 261602 [hep-ph/ 0501128 ].

[11] P. Colangelo, F. De Fazio, F. Giannuzzi, F. Jugeau and S. Nicotri, Light scalar mesons in the soft-wall model of AdS/QCD, Phys. Rev. D78 (2008) 055009 [0 807 . 10 54].

[12] Particle Data Group collaboration, Review of Particle Physics, Chin. Phys. C40 (2016) 100001.

[13] V. Vento, Scalar glueball spectrum, Phys. Rev. D73 (2006) 054006 [hep-ph / 0401218 ].

[14] V. Vento, Glueball-Meson Mixing, Eur. Phys. J. A52 (2016) 1 [1505. 05355].

[15] V. Mathieu, N. Kochelev and V. Vento, The Physics of Glueballs, Int. J. Mod. Phys. E18 (2009) 1 [0810.4453].

[16] Particle Data Group collaboration, Review of Particle Physics, Phys. Rev. D98 (2018) 030001.

[17] P. Colangelo, F. De Fazio, F. Jugeau and S. Nicotri, On the light glueball spectrum in a holographic description of QCD, Phys. Lett. B652 (2007) 73 [hep-ph/ 0703316 ].

[18] M. Rinaldi, V. Vento and R. Orava, Looking for pure glueball states, 1803.05738. 